\title{
Fast Bayesian Blind Deconvolution with Huber Super Gaussian Priors
}

\author{
Xu Zhou, Miguel Vega, Fugen Zhou, Rafael Molina and Aggelos K. Katsaggelos
}

\begin{abstract}
Expectation Maximization (EM) based inference has already proven to be a very powerful tool to solve blind image deconvolution (BID) problems. Unfortunately, three important problems still impede the application of EM in BID: the undesirable saddle points and local minima caused by highly nonconvex priors, the instability around zero of some of the most interesting sparsity promoting priors, and the intrinsic high computational cost of the corresponding BID algorithm. In this paper we first show how Super Gaussian priors can be made numerically tractable around zero by introducing the family of Huber Super Gaussian priors and then present a fast EM based blind deconvolution method formulated in the image space. In the proposed computational approach, image and kernel estimation are performed by using the Alternating Direction Method of Multipliers (ADMM), which allows to exploit the advantages of FFT computation. For highly nonconvex priors, we propose a Smooth ADMM (SADMM) approach to avoid poor BID estimates. Extensive experiments demonstrate that the proposed method significantly outperforms state-of-the-art BID methods in terms of quality of the reconstructions and speed.
\end{abstract}

\section{INTRODUCTION}

In the imaging process, factors like camera shake, defocus and atmospheric turbulence cause blurriness in the observed image. To remove the undesirable blur, one can use nonblind or blind deconvolution methods, depending on whether the blur is known or unknown, respectively. Blind image deconvolution (BID) is an interesting and challenging problem which finds application in areas like astronomical imaging, medical imaging, and computational photography [1].

In general, the degraded image $y$ can be approximately modelled as [2]

$$
y=H x+n
$$

where $x$ and $n$ are the original image and noise, respectively, and $H$ is the convolution matrix formed from the blur kernel $h$.

Given a single degraded image $y$, the BID goal is to estimate the original image $x$ and blur kernel $h$. BID is a severely ill-posed inverse problem, since there are infinitely many solutions for $x$ and $h$. To find the desirable ones from the set of uncountable solutions, additional assumptions on both $x$ and $h$ are indispensable. During the last decade, BID has been intensively studied in the image processing and computer vision communities [3]-[21]. It could be rightly claimed that the vast majority of state-of-the-art BID methods make use of sparsity promoting image priors. Most existing BID methods can be classified into three categories, that is, VB (variational Bayes), MAP (maximum a posterior), and other methods which we now briefly review. Notice that in this classification EM inference is included in the VB category.

Miskin and MacKay [3] assume a mixture of Laplacian priors for cartoon images and propose a VB approach, termed ensemble learning, to estimate the blur. Likas and Galatsanos [4] propose a similar VB approach with Gaussian priors, see also Molina et al. [22] for VB with Gaussian priors for image and blur. Fergus et al. [5] exploit

X. Zhou was with the Image Processing Center, Beihang University, Beijing 100191, China. He is now with ShanghaiTech University, Shanghai 200031, China, (e-mail: zhouxu179@163.com).

M. Vega and R. Molina are with Departamento de Ciencias de la Computación e I.A., Universidad de Granada, Granada 18071, Spain (e-mail: mvega@ugr.es; rms@decsai.ugr.es).

F. Zhou is with the Image Processing Center, Beihang University, Beijing 100191, China (e-mail: zhfugen@buaa.edu.cn).

A. Katsaggelos is with the Department of Electrical Engineering and Computer Science, Northwestern University, Evanston, IL 60208-3118 USA (e-mail: aggk@eecs.northwestern.edu).

This work was sponsored in part by National Natural Science Foundation of China (61233005), Ministerio de Ciencia e Innovación under Contract TIN2013-43880-R, the European Regional Development Fund (FEDER), the CEI BioTic with the Universidad de Granada and the Department of Energy grant DE-NA0002520. 
a mixture-of-Gaussians (MOG) prior and extend the VB approach [3] to handle natural images with nonparametric motion blurs. Levin et al. [10] also utilize an MOG prior on the latent image and propose a $M A P_{h}$ approach, namely marginalizing all latent images and estimating the kernel alone. Making use of the conjugate concave principle, Babacan et al. [13] propose a general Bayesian framework, which allows the use of general sparse priors for BID. This framework includes [5] and [10] as particular cases. Recently, following the $M A P_{h}$ approach [10] and assuming a Gaussian representation model, Zhang and Wipf [20] have proposed a BID method with parameter estimation to remove camera shake. Following the work in [13], Vega et al. [21] suggest introducing an additional parameter on the image prior and show that this parameter can be used to improve the quality of the restoration. Interested readers are referred to [2] and [23] for recent reviews on VB based BID methods.

Instead of imposing a prior distribution on the latent image, one can also use shock filters [24] to promote sparsity, see [7], [8] and [16]. The shock filter provides a highly smooth image with predicted sharp step edges, which can be used as a reference image to regularize the latent image. Since the predicted step edges are probably inaccurate, a better approach is to use nondimensional sparsity measures [11] [19] [17], which model ideal step edges as local minima. Sun et al. [14] propose a parametric step edge model to promote sparsity, where the parameters are learned from synthetic or natural image patches. Recently, Perrone and Favaro [25] have proposed a logarithmic TV (Total Variation) based BID methods using a delayed kernel normalization scheme [26]. The above MAP methods seek a cartoon like image to estimate the blur kernel and hence are robust to noise; however they may fail if the original image lacks step edges or is dominated by textures.

While most of the existing BID methods focus on image priors, priors on the kernel have received much less attention. Cai et al. [9] suggest using curvelet representations for motion blurs, since the curvelet representation takes into account both the continuity and sparsity of motion blur kernels. Goldstein and Fattal [12] propose a spectrum power prior on the blur kernel, based on the assumption that the spectrum power of a natural image drops quadratically as the frequency increases. Zhou et al. [27] propose a variational Dirichlet approach to estimate the blur kernel and show that it helps reduce noise in the estimated kernel.

Although Super Gaussian (SG) [13] distributions have proven to be the right tool to introduce prior information in BID, some of the most widely used such distributions, like $\ell_{p}$ and log priors, suffer from lack of differentiability around zero. In this paper we introduce in a sound way a new family of SG distributions fully differentiable and thus well behaved around zero. We call them Huber Super Gaussian (HSG) priors.

As is widely known, VB based BID methods involve two major steps: image and kernel estimation. They correspond to solving large-scale linear equation problems and providing solution to large scale quadratic problems with constraints, respectively. In this work, by incorporating the latest techniques in image deconvolution [13] [28], we also present a fast Bayesian blind deconvolution method formulated in the image space for Huber Super Gaussian priors.

Recent advances in BID [13] [20] [17] [23] [25] [27] show that highly nonconvex priors are very effective in BID problems. However, the local minima and saddle point problem caused by nonconvex priors, e.g., log prior, has not been well addressed. We report that the EM approach, which provides an estimate of the mean of the posterior distribution of the latent image and has been used in Bayesian BID methods [10] [13] [20], often leads to a stationary point with a large objective function value and an image estimate which is not adequately smooth. We show that, with suitable parameter settings and iterative schemes, ADMM can be used to tackle this problem. The proposed ADMM with a large penalty weight finds a smooth approximate solution to the reweighted linear equations, rather than the exact solution as searched by the EM approach. Experiments show that the proposed ADMM, which seeks a smooth image mean rather than the exact image mean provided by the EM approach, leads to a smoother image with a lower cost value, and the proposed BID method significantly outperforms state-of-the-art BID methods in terms of restoration quality and speed.

In summary, the contributions of this paper are a) the introduction of the Huber Super Gaussian prior family to improve numerical stability and convergence speed, and b) a fast method for nonconvex image deconvolution, which avoids undesirable stationary points and yields comparable or even better results than the EM approach in terms of ringing artifact reduction.

The paper is organized as follows. In section II we formulate the BID problem. We introduce the observation model, define the family of Huber Super Gaussian priors and present the inference framework. Section III] is devoted to the image and blur reconstruction algorithms. Section IV discusses, in detail, all the parameters involved in the BID algorithm we propose. Section $\mathrm{V}$ compares our approach with current state-of-the-art BID algorithms, and 


\begin{tabular}{|c|}
\hline$\rho(s)$ \\
\hline$\frac{1}{2} s^{2}$ \\
\hline$\frac{1}{p}|s|^{p}$ \\
\hline $\log |s|$ \\
\hline$-\log \sum_{j} \frac{\pi_{j}}{\sqrt{2 \pi} \sigma_{j}} \exp \left(-\frac{s^{2}}{2 \sigma_{j}^{2}}\right)$ \\
\hline
\end{tabular}

(a)

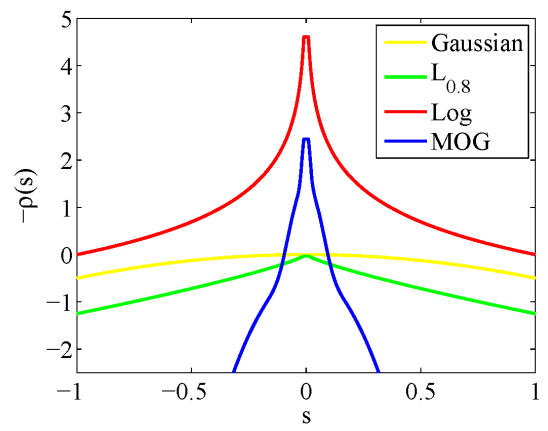

(b)

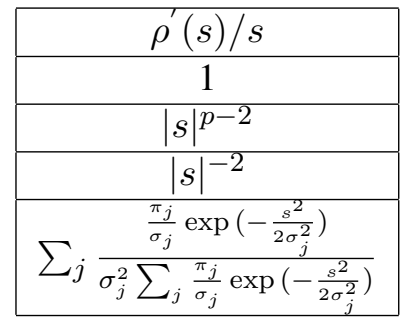

(c)

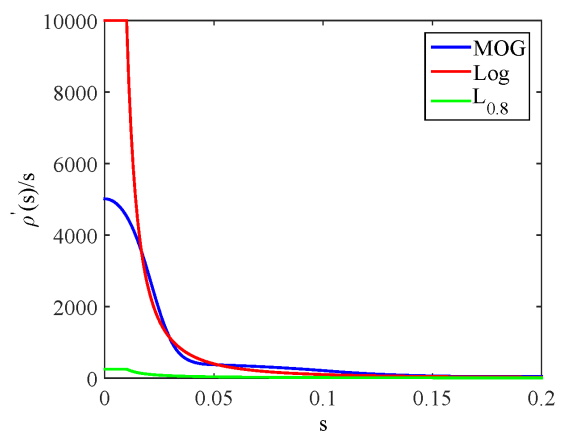

(d)

Fig. 1. (a) and (b): examples of penalty functions $\rho(s)$, where the MOG is obtained from Levin et al. $|10|$. (c): their corresponding $\rho^{\prime}(s) / s$. (d) Plots of $\rho_{\epsilon}^{\prime}(s) / s$, where $\epsilon=0.01$ for $\log$ and $\mathrm{L}_{0.8}$.

section $\mathrm{VI}$ concludes the paper.

\section{PRIOR MODEL}

Let the sizes of $y, x$, and $h$ in Eq. $(1)$ be $\sqrt{N} \times \sqrt{N}, \sqrt{N} \times \sqrt{N}$ and $\sqrt{K} \times \sqrt{K}$, respectively. We assume that $H$ is block circulant with circulant blocks, and then have $H=\mathcal{F}^{-1} \operatorname{diag}(\mathcal{H}) \mathcal{F}$, where $\mathcal{F}$ denotes the 2-D DFT and $\mathcal{H}=\mathcal{F} P h$ is the Fourier transform of $h$, with $P$ being an $N \times K$ matrix that pads $h$ with zeros to the size $\sqrt{N} \times \sqrt{N}$. The dimensions of $H$ and $\operatorname{diag}(\mathcal{H})$ are therefore $N \times N$. For simplicity, we overload the notations $x$, $y$, and $h$ with two meanings, a 2-D array or a column vector arranged in lexicographic order; which notation is being used will be clear from the context.

Assuming an i.i.d. Gaussian noise with variance $\sigma^{2}$, we can write

$$
p(y \mid x, h)=\frac{1}{(\sqrt{2 \pi} \sigma)^{N}} \exp \left(-\frac{\|H x-y\|_{2}^{2}}{2 \sigma^{2}}\right)
$$

Let us now proceed to define the image prior model we use in the paper by introducing first its energy (penalty) function.

Let $\rho(s): \Re \rightarrow[-\infty,+\infty]$ be symmetric around zero with $\rho(\sqrt{s})$ concave and increasing for $s \in[0, \infty)$. This condition is equivalent to $\rho^{\prime}(s) / s$ being decreasing on $(0,+\infty)$, that is, for $s_{1} \leq s_{2}, \rho^{\prime}\left(s_{1}\right) / s_{1} \geq \rho^{\prime}\left(s_{2}\right) / s_{2}$. A probability distribution of the form $p(s) \propto \exp (-\rho(s))$ is called a Super Gaussian (SG) distribution. SG distributions promote sparsity. Their use in blind image restoration problems was first proposed in [13]. Examples of energies associated to SG distributions are shown in Figs. 1 (a) and 1 (b), in which $\rho(s)=\log (|s|)$, the so called $\log$ prior, was first introduced in [13].

The energy, $\rho(\cdot)$, associated to an SG distribution can be represented as (see [29])

$$
\rho(s)=\inf _{\xi>0} \frac{1}{2} \xi s^{2}-\rho^{*}\left(\frac{1}{2} \xi\right)
$$

where $\rho^{*}(\xi / 2)$ is the concave conjugate function

$$
\rho^{*}\left(\frac{1}{2} \xi\right)=\inf _{s} \frac{1}{2} \xi s^{2}-\rho(s) .
$$


Furthermore, as shown in [13], the infimum in 3 is achieved at $\xi=\rho^{\prime}(s) / s$.

Equation (3) provides a quadratic upper bound to the energy of an SG distribution which naturally leads to a Gaussian approximation. Unfortunately, for very useful energies associated to SG distributions, like $\rho(s)=|s|^{p} / p$ $(0<p<1)$ and $\rho(s)=\log (|s|), \rho(s)$ is not differentiable at zero and $\xi=\rho^{\prime}(s) /|s|$ is unbounded as $s$ approaches zero. We overcome this problem by defining here for $\epsilon>0$ the robust symmetric around zero function $\rho_{\epsilon}(s)$ associated with $\rho(s)$ as follows

$$
\rho_{\epsilon}(s)= \begin{cases}\rho(s) & \text { for } s \geq \epsilon \\ \frac{\rho^{\prime}(\epsilon)}{2 \epsilon} s^{2}-\left(\frac{\rho^{\prime}(\epsilon)}{2} \epsilon-\rho(\epsilon)\right) & \text { for } 0 \leq s \leq \epsilon\end{cases}
$$

Furthermore, since $\rho^{\prime}(s) / s$ is decreasing on $(0, \infty)$,

$$
\frac{\rho_{\epsilon}^{\prime}(s)}{s}=\min \left(\rho^{\prime}(s) / s, \rho^{\prime}(\epsilon) / \epsilon\right)= \begin{cases}\frac{\rho^{\prime}(s)}{s} & \text { for } s \geq \epsilon \\ \frac{\rho^{\prime}(\epsilon)}{\epsilon} & \text { for } 0 \leq s \leq \epsilon\end{cases}
$$

and so $\rho_{\epsilon}(s)$ is also the energy of an SG distribution.

For the $\log$ and $\ell_{p}$ priors mentioned above we have $\rho_{\epsilon}^{\prime}(s) / s=\min \left(s^{-2}, \epsilon^{-2}\right)$ and $\rho_{\epsilon}^{\prime}(s) / s=\min \left(s^{p-2}, \epsilon^{p-2}\right)$, respectively, see Fig. 11(d). The use of $\rho_{\epsilon}(s)$ makes $\rho(s)$ differentiable at zero and the prior tractable. Following the terminology in [30], we call these priors Huber Super Gaussian (HSG) priors. We defer the analysis of this prior to section IV.

Having defined the robust energy associated to an HSG distribution, we now proceed to introduce the image model we use. The prior $p(x)$ on the latent image has the form

$$
p(x)=Z \exp \left(-\sum_{\gamma=1}^{L} \sum_{i} \rho_{\epsilon}\left(x_{\gamma}(i)\right)\right),
$$

where $x_{\gamma}=f_{\gamma} \otimes x$ with $\otimes$ denoting a circular convolution operator and $\left\{f_{\gamma}\right\}$ being a set of $L$ high pass filters. We will discuss the filters used in this work in the experimental section.

For the blur we do not assume any prior knowledge apart from the fact that it must be nonnegative and its coefficients should add to one. That is, $p(h) \propto$ const, subject to: $h(i) \geq 0, \sum_{i} h(i)=1$.

\section{BAYESIAN INFERENCE}

The process to estimate the blur and image will be carried out as follows. First, we estimate the blur using the EM approach to solve

$$
\begin{aligned}
\hat{h}= & \arg \max _{h} \int p(x, h, y) d x \\
& \text { s.t. } h(i) \geq 0, \sum_{i} h(i)=1,
\end{aligned}
$$

and then once the blur has been estimated we use it to estimate the original image utilizing a nonblind image deconvolution algorithm. Notice that, in principle, we should use $p(x \mid \hat{h}, y)$ to estimate the original image. However, our sparsity promoting image prior based on $\rho_{\epsilon}(\cdot)$ is designed to obtain a good blur estimate when the joint distribution is marginalized on the image at the cost of removing fine details in the image estimate.

Directly applying EM inference on $p(x, h, y)$ to estimate the blur is infeasible due to the form of $p(x)$. Since $\rho_{\epsilon}(s)$ is the energy associated to an SG distribution, we can write

$$
p(x) \geq Z \prod_{\gamma=1}^{L} \prod_{i} \exp \left(-\left(\frac{\xi_{\gamma}(i)}{2} x_{\gamma}^{2}(i)-\rho_{\epsilon}^{*}\left(\frac{1}{2} \xi_{\gamma}(i)\right)\right)\right), \forall \xi_{\gamma}(i)>0 .
$$

This Gaussian-like lower bound allows for the expectation of the joint distribution to be calculated analytically. We have

$$
p(x, h, y) \geq p(y \mid x, h) p(h) Z e^{-\sum_{\gamma=1}^{L} \sum_{i}\left(\frac{\xi_{\gamma}(i)}{2} x_{\gamma}^{2}(i)-\rho_{\epsilon}^{*}\left(\frac{1}{2} \xi_{\gamma}(i)\right)\right)} \equiv Q(y, x, h, \boldsymbol{\xi}),
$$

where $\boldsymbol{\xi}=\left\{\xi_{\gamma}(i), \gamma=1, \ldots, L, i=1, \ldots, N\right\}$ with all components positive. 
We finally obtain

$$
\begin{aligned}
\hat{h} & =\arg \max _{h} E[\log (Q(y, x, h, \hat{\boldsymbol{\xi}}))]_{\hat{q}(x)}, \\
\hat{q}(x) & \propto Q(y, x, \hat{h}, \hat{\boldsymbol{\xi}}), \\
\hat{\boldsymbol{\xi}} & =\arg \max _{\boldsymbol{\xi}>0} E[\log (Q(y, x, \hat{h}, \boldsymbol{\xi}))]_{\hat{q}(x)} .
\end{aligned}
$$

Notice that $\hat{q}(x)$ is an approximation of the posterior distribution $p(x \mid \hat{h}, y)$.

\section{A. Estimation of image and variational parameter}

For the latent image, we obtain from $[13$, ,

$$
\log \hat{q}(x)=-\frac{1}{2 \sigma^{2}}\|\hat{H} x-y\|_{2}^{2}-\frac{1}{2} \sum_{\gamma=1}^{L} x_{\gamma}^{T} \operatorname{diag}\left(\hat{\xi}_{\gamma}\right) x_{\gamma},
$$

which is a multivariate Gaussian with precision matrix

$$
C_{x}^{-1}=\frac{1}{\sigma^{2}} \hat{H}^{T} \hat{H}+\sum_{\gamma=1}^{L} F_{\gamma}^{T} \operatorname{diag}\left(\hat{\xi}_{\gamma}\right) F_{\gamma},
$$

where $F_{\gamma}$ is an $N \times N$ circulant convolution matrix formed by the kernel $f_{\gamma}$. The mean $\hat{x}$ is used as the estimate for $x$, which is obtained by solving the following linear system of equations

$$
C_{x}^{-1} \hat{x}=\frac{1}{\sigma^{2}} \hat{H}^{T} y
$$

This linear system can be solved by ADMM [28] very efficiently, as we will show in the following.

For the variational parameter $\hat{\xi}$, we obtain from 14

$$
\left.\left.\hat{\xi}_{\gamma}(i)=\frac{\rho_{\epsilon}^{\prime}\left(\nu_{\gamma}(i)\right)}{\nu_{\gamma}(i)}=\min \left(\rho^{\prime}\left(\nu_{\gamma}(i)\right)\right) / \nu_{\gamma}(i)\right), \rho^{\prime}(\epsilon) / \epsilon\right)
$$

where $\nu_{\gamma}(i)=\sqrt{E\left[x_{\gamma}^{2}(i)\right]}, 1 \leq i \leq N$, and the expected value is easily calculated using the distribution $\hat{q}(x)$ and the approximation similar to [10] [13]

$$
C_{x} \approx\left(\operatorname{diag}\left(C_{x}^{-1}\right)\right)^{-1}
$$

Given a kernel estimate, we need to alternatively solve the linear system (17) and update the weights (18). This "joint" estimation of the image and weights is understood as a standard nonblind image deconvolution procedure. It proceeds as an IRLS (Iterative Reweighted Least Squares) frequently used in sparse signal recovery problems [28]. Previous methods [10] [13] [20] use the Conjugate Gradient (CG) algorithm to solve (17), which is very time consuming. Instead of using $\mathrm{CG}$, we use ADMM to solve (17). The core idea is to treat the solution of a linear system as the minimizer of a quadratic function (see [28]). We transform the unconstrained problem into a constrained one by introducing the auxiliary variables, $v_{\gamma}=f_{\gamma} \otimes x[31]$. Consequently, for a given blur $H, \hat{x}$ can be rewritten as the minimizer of

$$
\begin{aligned}
f\left(x, v_{\gamma}\right)= & \frac{1}{2}\|H x-y\|_{2}^{2}+\sigma^{2} \sum_{\gamma=1}^{L} v_{\gamma}^{T} \operatorname{diag}\left(\hat{\xi}_{\gamma}\right) v_{\gamma} \\
& \text { s.t. } v_{\gamma}=F_{\gamma} x,
\end{aligned}
$$

which can be solved efficiently using ADMM. To be precise, we form the augmented Lagrangian

$$
\mathcal{L}_{\beta_{v}}\left(x, v_{\gamma}, d v_{\gamma}\right)=\frac{1}{2}\|H x-y\|_{2}^{2}+\sigma^{2} \sum_{\gamma=1}^{L} v_{\gamma}^{T} \operatorname{diag}\left(\hat{\xi}_{\gamma}\right) v_{\gamma}+\frac{\beta_{v}}{2} \sum_{\gamma=1}^{L}\left\|v_{\gamma}-F_{\gamma} x+d v_{\gamma}\right\|_{2}^{2}
$$




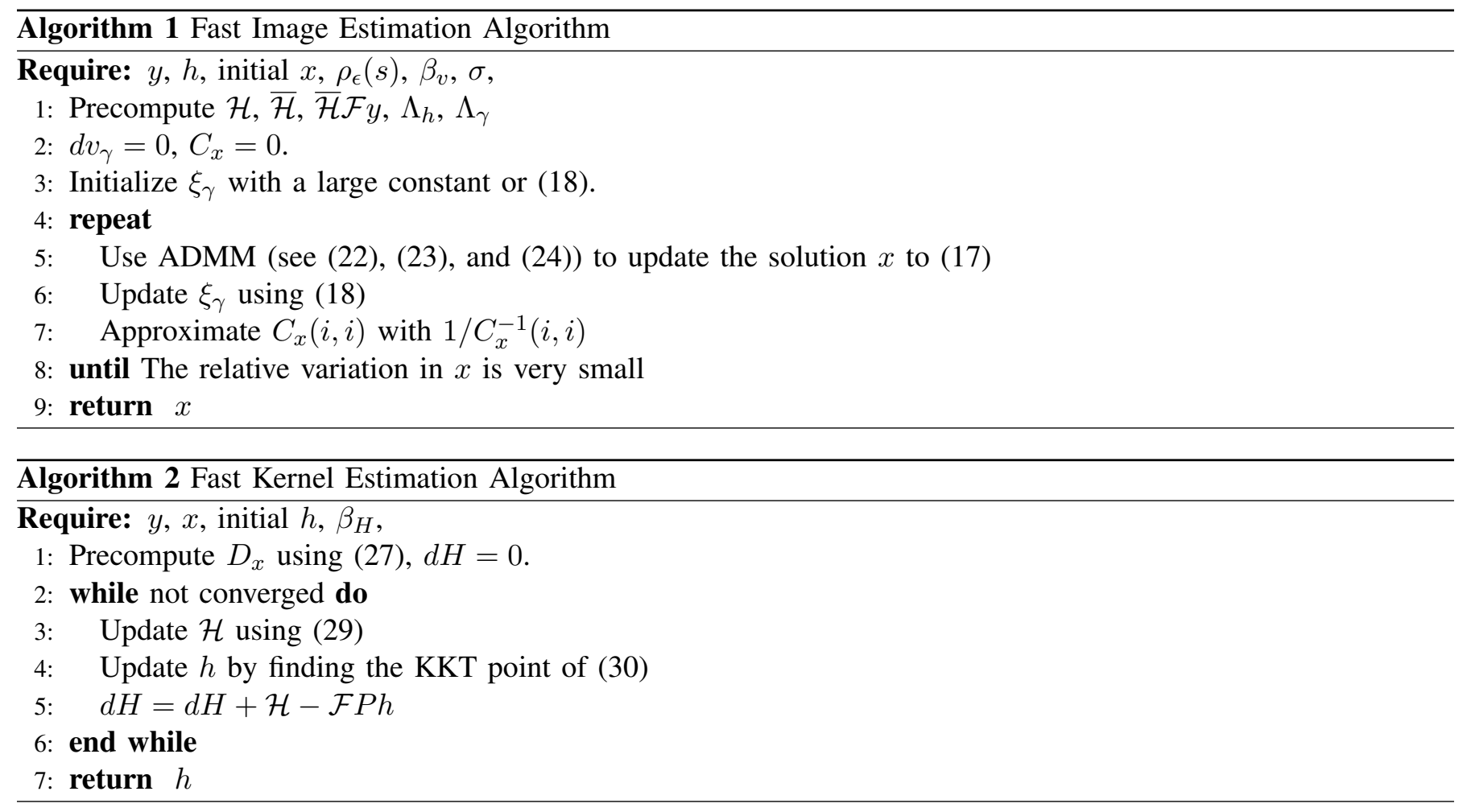

where $\beta_{v}$ is the penalty weight which enforces the constraints in 20 and controls the convergence rate, $d v_{\gamma}$ are the (scaled) dual variables (a.k.a., Lagrangian multipliers). ADMM consists of the iterations

$$
\begin{aligned}
x & =\mathcal{F}^{-1}\left\{\frac{\overline{\mathcal{H}} \mathcal{F} y+\beta_{v} \mathcal{F}\left[\sum_{\gamma=1}^{L} F_{\gamma}^{T}\left(v_{\gamma}+d v_{\gamma}\right)\right]}{\Lambda_{h}+\beta_{v} \sum_{\gamma=1}^{L} \Lambda_{\gamma}}\right\}, \\
v_{\gamma} & =\frac{\beta_{v}}{\beta_{v}+\sigma^{2} \hat{\xi}_{\gamma}}\left(F_{\gamma} x-d v_{\gamma}\right), \\
d v_{\gamma} & =d v_{\gamma}+v_{\gamma}-F_{\gamma} x,
\end{aligned}
$$

where $\overline{\mathcal{H}}$ is the complex conjugate of $\mathcal{H}$, and $\Lambda_{h}$ and $\Lambda_{\gamma}$ are the eigenvalues of $H^{T} H$ and $F_{\gamma}^{T} F_{\gamma}$, respectively.

The final algorithm for image estimation is presented in Alg. 1, which achieves state-of-the-art performance in terms of quality of restorations as well as speed. It should be noted that the image estimation problem is not trivial when $\rho(s)$ is nonconvex. For instance, if we choose the log prior, it is shown in [28] that Alg. 1 will converge to a stationary point of the cost function, which might be a saddle point or a local minimum. Therefore, the optimization scheme makes a considerable difference to the final reconstruction.

Before concluding this section we would like to mention that if $\nu_{\gamma}(i)=\sqrt{E^{2}\left[x_{\gamma}(i)\right]}\left(1 \leq i \leq N, C_{x}=0\right)$, then our procedure would not take into account the uncertainty of the image estimate and we would have a MAP procedure.

\section{B. Estimation of blur}

To estimate the blur we have

$$
\begin{gathered}
\hat{h}=\arg \min _{h}\|H \hat{x}-y\|_{2}^{2}+h^{T} D_{x} h \\
\text { s.t. } h(i) \geq 0, \sum_{i} h(i)=1,
\end{gathered}
$$

where $D_{x}$ is the $K \times K$ matrix given by

$$
D_{x}(m, n)=\sum_{j=1}^{N} C_{x}(m+j, n+j) .
$$


Given an image estimate, we use ADMM to efficiently solve the quadratic optimization problem (25) with nonnegativity and normalization constraints [26). By introducing the constraint $\mathcal{H}=\mathcal{F} P h$ (see [18] for a different variable splitting), we find $\hat{h}$ in 25 as the minimizer of

$$
\begin{aligned}
g(h, \mathcal{H})= & \|\mathcal{H} \circ \mathcal{X}-\mathcal{F} y\|_{2}^{2}+h^{T} D_{x} h \\
\text { s.t. } & h(i) \geq 0, \sum_{i} h(i)=1, \\
& \mathcal{H}=\mathcal{F} P h,
\end{aligned}
$$

where $\mathcal{X}=\mathcal{F} x$ denotes the Fourier transform of $x$ and $\circ$ element-wise multiplication. The ADMM algorithm for this constrained problem is presented in Alg. 2, in which the updates for $\mathcal{H}$ and $h$ are given by

$$
\begin{aligned}
\mathcal{H}= & \frac{\overline{\mathcal{X}} \circ(\mathcal{F} y)+\beta_{H}(\mathcal{F} P h-d H)}{\overline{\mathcal{X}} \circ \mathcal{X}+\beta_{H}} \\
h= & \arg \min _{h}\left\|h-P^{T} \mathcal{F}^{-1}(\mathcal{H}+d H)\right\|_{2}^{2}+h^{T} D_{x} h \\
& \text { s.t. } h(i) \geq 0, \sum_{i} h(i)=1
\end{aligned}
$$

where the $L_{2}-$ norm term in 30 is obtained by observing that $\forall h \in \Re^{K}$, the following equalities hold $\|\mathcal{F} P h\|_{2}^{2}=$ $\|h\|_{2}^{2}=\left\|P^{T} \mathcal{F}^{-1} \mathcal{H}\right\|_{2}^{2}$.

The convex problem (30) can be solved by the Karush-Kuhn-Tucker (KKT) conditions, which refers to solving a system of nonlinear equations. Specifically, we use a fixed point iteration method to find the KKT point. It should be noted that the computation complexity of solving problem 30 is only $O(K)$ rather than $O\left(K^{2}\right)$, since $D_{x}$ is approximated by a diagonal matrix.

The proposed ADMM splits the constrained large scale quadratic program into two simpler subproblems: one which can be solved directly in Fourier domain and another which is a small scale quadratic program that can be solved iteratively. Moreover, with 10 ADMM iterations, a very good approximate solution can be obtained. In most cases, we observed that only 5 ADMM iterations are needed for convergence.

\section{Multiscale blind deconvolution}

As with previous BID methods [5], [10], [13], we also adopt here a multiscale approach to estimate the kernel. Fergus et al. [5] point out that single scale BID may suffer from local minima, especially when the support of the kernel is large. The multiscale approach, however, is very useful for large support kernel estimation. The rational behind this approach is that at the coarsest level, the blur is alleviated significantly, so that it is easier to estimate a kernel from the downsampled image. At the next finer level, this kernel estimate is upsampled and can be used as a good starting point for the single scale BID. Repeating this process until the finest level, we obtain a better kernel estimate.

\section{Final image reconstruction}

The estimated image $\hat{x}$ obtained above is appropriate for estimating the blur but, as we have already explained and also as we will show in the experimental section, it is over-smooth. This is because the sparsity promoting weight $\sigma^{2} \xi_{\gamma}$ is too large to recover the fine details, especially in the case of the log prior. To recover more details, we reconstruct the final sharp image $x^{*}$ by solving the following problem [32] [33],

$$
x^{*}=\arg \min _{x} \frac{1}{2}\|H x-y\|_{2}^{2}+\frac{\lambda_{x}}{p} \sum_{\gamma}\left\|f_{\gamma} \otimes x\right\|_{p},
$$

where $\left\{f_{\gamma}\right\}$ is a set of derivative filters to be defined in the experimental section, $p$ is typically in the range $[0.6,0.8]$ [32] and the $\ell_{p}$ regularizer should be understood in the HSG form. 


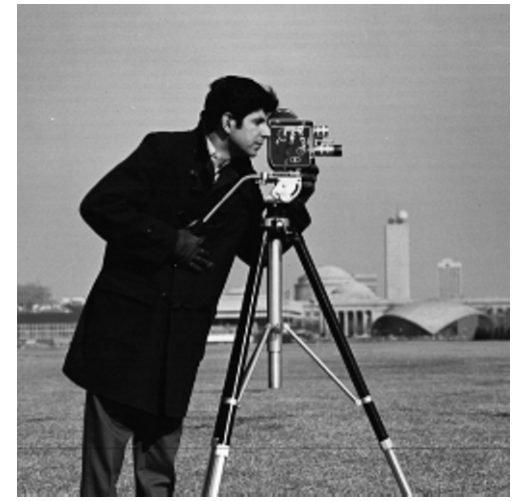

(a) Groundtruth

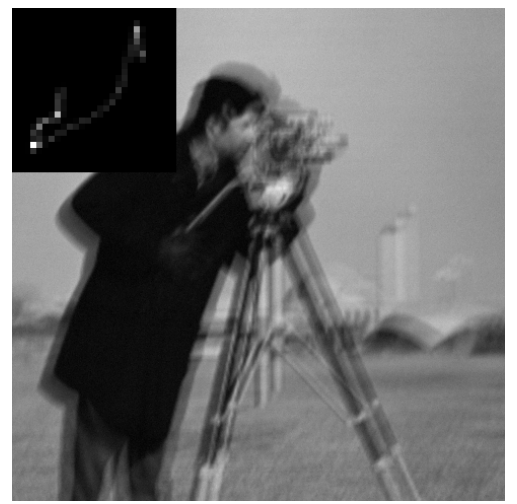

(d) Input

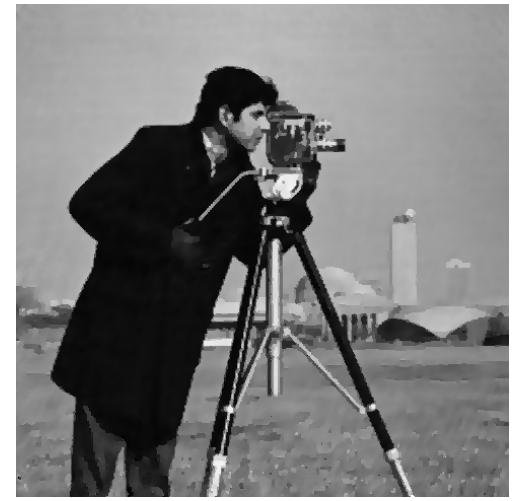

(b) MAP (ADMM)

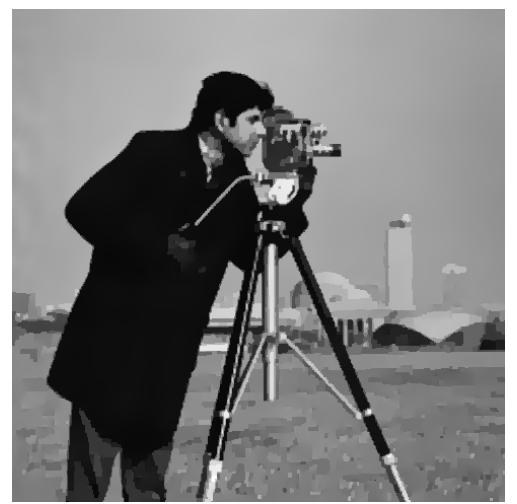

(e) MAP (SADMM)

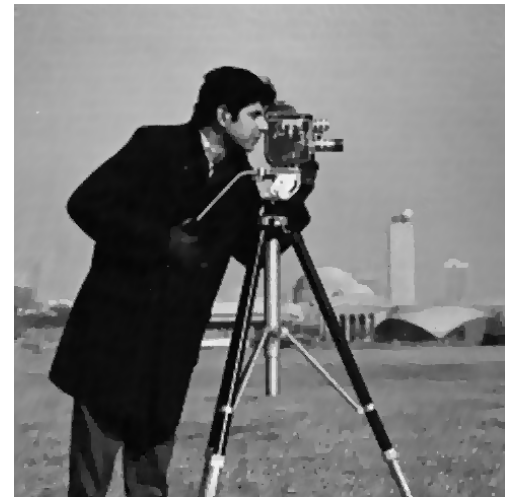

(c) EM (ADMM)

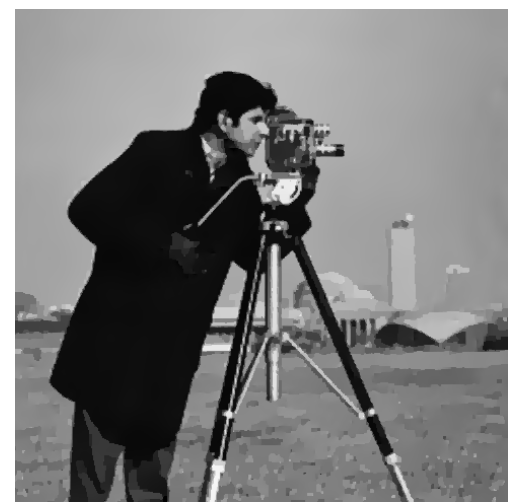

(f) EM (SADMM)

Fig. 2. SADMM vs. ADMM in nonblind deconvolution: restorations. (a) Groundtruth. (b) Restoration by MAP (ADMM). (c) Restoration by EM (ADMM). (d) Degraded image with groundtruth kernel, noise level $\sigma=0.01$. (e) Restoration by MAP (SADMM). (f) Restoration by EM (SADMM).

\section{E. Summary}

So far we have presented a fast EM variational Bayesian approach for blind image deconvolution formulated in the image space, using a lower bound on the HSG image priors.

The benefit of working in the image space rather than in the filter space [5] [10] [13] is threefold. First, utilizing the image space can save computations by nearly $100 \times \frac{L-1}{L} \%$, compared to the filter space. Second, the image space is less sensitive to noise, since using the filter space amplifies the noise in the observations. Third, the filter space solution ignores the important integrability constraint [10], whereas the image space solution does not suffer from this problem.

The proposed BID method, like many existing BID methods [5] [8] [11] [10] [13] [19] [17], just to name a few, alternatively iterates between two major steps, image estimation with the current kernel estimate and kernel estimation with the current image estimate. The image estimation step requires to solve the large scale linear system (17) and update the weights $\xi$ (18). By estimating the image and variational parameters jointly, the image estimation step is a standard nonblind deconvolution procedure.

The weights $\boldsymbol{\xi}$ (variational parameters) play an important role in sparse recovery problems. In fact, large values in $\xi$ determine which pixels should be smoothed, and therefore help remove the blur, provided that the location is correct (consistent with the groundtruth).

\section{Parameter Analysis of Alg. 1}

In this section, we analyze the key parameters of Alg. 11, such as the number of ADMM iterations, $\beta_{v}, \epsilon$, and $\sigma^{2}$. Throughout this section and what follows, EM stands for the procedure described to estimate image, weights, and blur. Notice that we use ADMM to solve (17). 


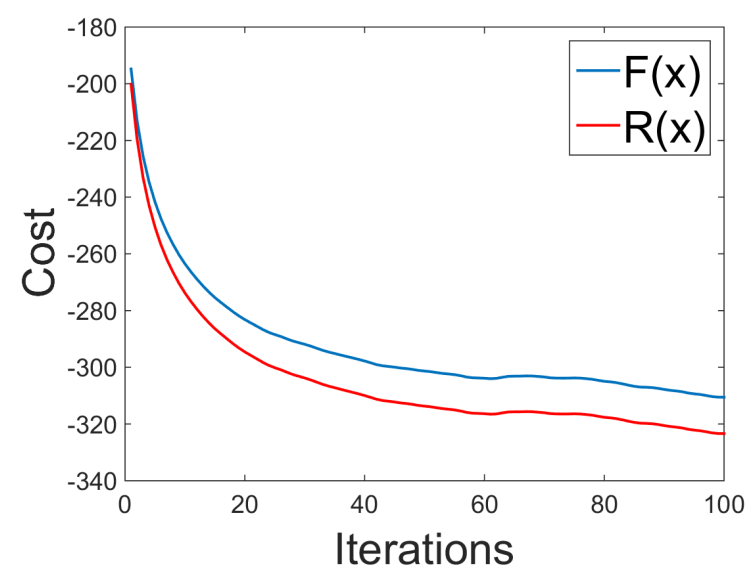

(a) MAP (ADMM)

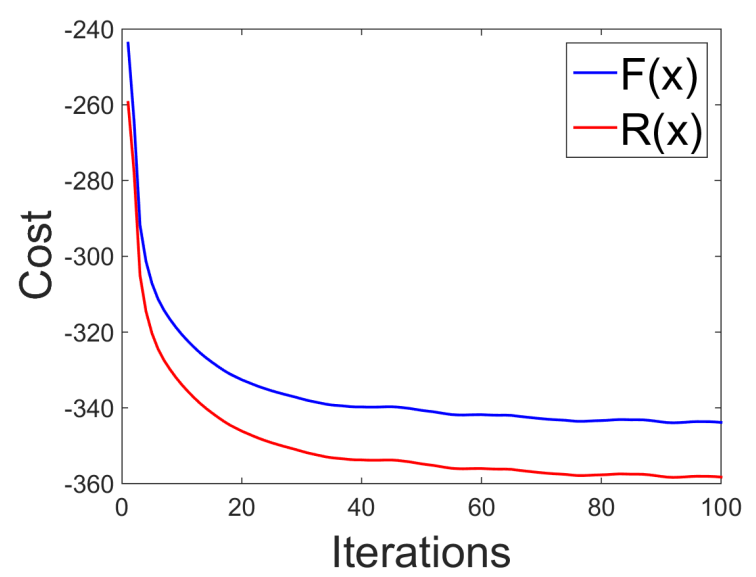

(c) MAP (SADMM)

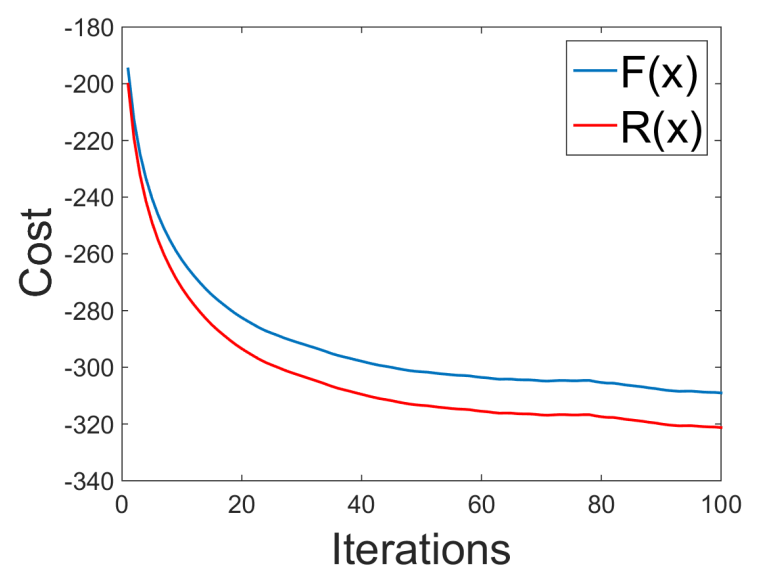

(b) EM (ADMM)

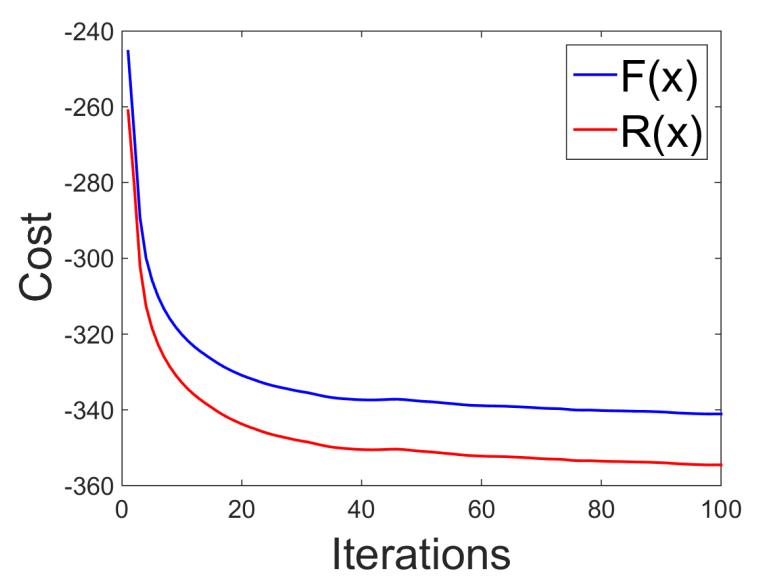

(d) EM (SADMM)

Fig. 3. SADMM vs. ADMM in nonblind deconvolutions: (a) Cost profile by MAP (ADMM); (b) Cost profile by EM (ADMM); (c) Cost profile by MAP (SADMM); (d) Cost profile by EM (SADMM).

Regarding the number of ADMM iterations, for the Step 5 of Alg. 1. we observe that 4 ADMM iterations with a small penalty weight $\left(\beta_{v}=0.001\right)$ is sufficient to find a good approximate solution to (17). However, it turns out that finding such an accurate solution to $(17)$ often leads to poor local minima or saddle points in the sense of the objective function value. Instead of solving (17) fully, we solve (17) partially and use a large penalty weight $\beta_{v}$ to find a smooth approximate solution to (17). Unfortunately, large $\beta_{v}$ reduces the convergence speed [31] [34]. We therefore use only one ADMM iteration and update the weights more frequently for acceleration [28]. In the performed experiments, we have observed that appropriate images to estimate the blur are those with many step edges and tiny sharp details while not suitable images to perform the same task are those which are not smooth and also contain ringing artifacts. Based on this observation, we use a large penalty weight $\beta_{v}$ to enforce strong image smoothness during the optimization procedure, so that ringing or blur is less likely to emerge. In fact, the denominator of Eq. (22) indicates that large $\beta_{v}$ makes the high frequency components of $\Lambda_{h}$ well regularized, so that ringing is suppressed even if $\Lambda_{h}$ is not accurate. We call this approach Smooth ADMM (SADMM) because it does not provide the exact EM solution to equation (17) but a smoother image estimate that can be used to better estimate the blur.

We should mention that one ADMM iteration for Step 5 cannot guarantee that the cost function drops monotonically. For this to happen, more ADMM iterations are required for Step 5. Interested readers are referred to Fig. 2(e) in [28], for a challenging nonconvex image reconstruction for which the cost function does not drop monotonically but still converges after thousands of iterations. Notice that we can not prove the convergence of Alg. 1 with one ADMM iteration, however we have not experienced any convergence problems in the performed experiments. 


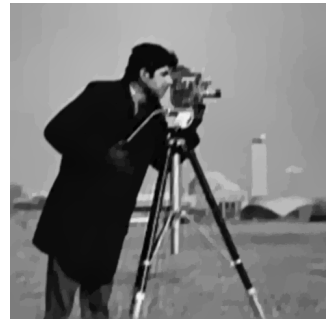

(a)

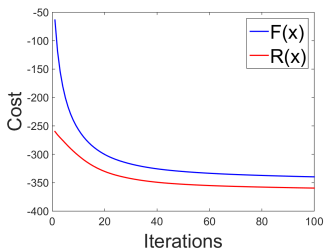

(f)

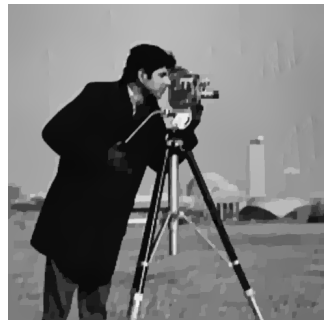

(b)

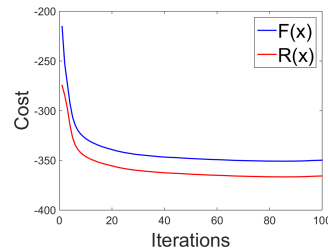

(g)

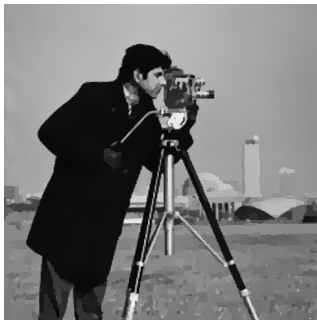

(c)

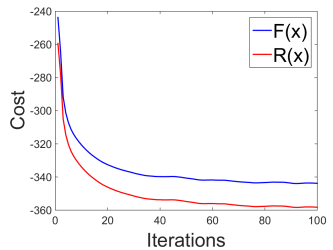

(h)

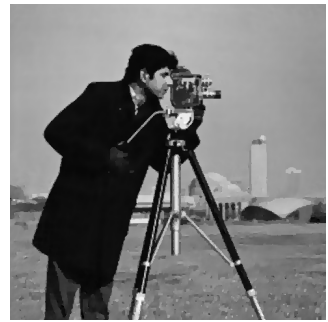

(d)

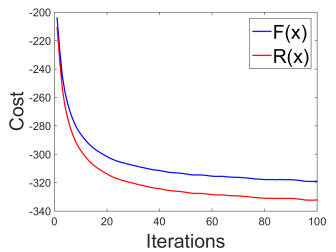

(i)

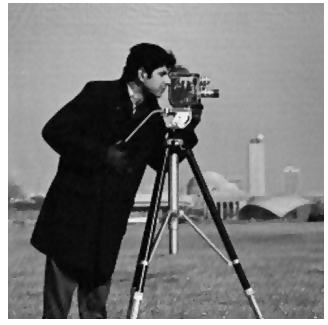

(e)

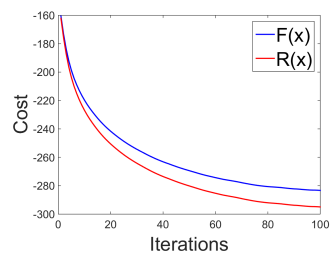

(j)

Fig. 4. Restorations with different $\beta_{v}$. (a) $\beta_{v}=10$. (b) $\beta_{v}=1$. (c) $\beta_{v}=0.1$. (d) $\beta_{v}=0.01$. (e) $\beta_{v}=0.001$. (f)-(j) are the cost profiles of (a)-(e), respectively.

To demonstrate the advantages of SADMM (fewer ADMM iterations, $100 \times 1$, and a large $\beta_{v}$ equal to 0.1 ) over $\mathrm{EM}$ (more $\mathrm{ADMM}$ iterations, $100 \times 4$, and a small $\beta_{v}$ equal to 0.001 ), we adopt the $\log$ prior and test the SADMM and ADMM in nonblind image deconvolution using the synthetic degradation in Fig. 2(a), whose groundtruth image is shown in Fig. 2 (d). We use the MAP cost function, that is, $F(x)=\frac{1}{2}\|H x-y\|_{2}^{2}+R(x)$, where $R(x)=\sigma^{2} \sum_{i} \sum_{\gamma=1}^{L} \log _{\epsilon}\left(\left|x_{\gamma}(i)\right|\right)$ and $\log _{\epsilon}(\epsilon=0.001)$ is the Huber $\log$ function. Fig. 2 (b) shows the recovered image by MAP (ADMM), which is not very smooth and contains a small amount of boundary artifacts. In contrast, the restoration by MAP (SADMM) (Fig. 2(e)) is smoother and with many sharp step edges, including the grass region. The cost functions, presented in Figs. 3(a) and (c), indicate that the proposed SADMM converges faster than the ADMM. It should be noted that ADMM needs 800 FFTs (including 400 IFFTs) to generate such a smooth restoration 1 , whereas SADMM requires only 200 FFTs (including 100 IFFTs). The same conclusions can also be drawn for the EM case, as shown in Figs. 2(c), 2(f), 3(b), and 3(d).

We now analyze the penalty weights for the ADMM procedure. Figure 4 shows the impact of $\beta_{v}$ on the SADMM results, for the input image in Fig. 2(a). It is clear that the recovered image becomes smoother as $\beta_{v}$ increases. However, if $\beta_{v}$ is too large, it can not recover sufficient (sharp) image edges and the convergence speed becomes slow (the image evolves slowly in each iteration), as shown in Figs. 4(a) and (f), respectively. Fig. 4(c) suggests that $\beta_{v} \in[0.1,1]$ is a good choice for Alg. 1, since it can enforce adequately strong smoothness and also recover many sharp step edges. In short, $\beta_{v}$ controls the image smoothness and convergence speed.

Regarding the robustness parameter $\epsilon$ used in the prior, we note that it plays an important role on the image sparsity as well as the numerical stability of the estimation procedure. The HSG prior favors an image of smaller sparsity than the SG prior, depending on the value of $\epsilon$. From (10) and (18), it is clear that increasing $\epsilon$ promotes less sparsity, since the weights $\boldsymbol{\xi}$ will be upper bounded by a smaller value. Figure 5 shows the restorations with 5 different values of $\epsilon$ ranging from 0 to 0.02. As we can see in Figs. 5 (a)-(e), the strong edges are basically the same, however the weak edges are different. As $\epsilon$ increases, more weak edges appear and the image gets somewhat blurry, see the selected patches in Figs. 5(d) and (e). Figures 5(a) and (b) are quite similar, suggesting that a small $\epsilon$ like 0.002 is enough to promote very strong sparsity for image deblurring. This figure shows that a small $\epsilon$ is crucial for a smooth and blur free restoration. However, as $\epsilon$ drops to zero, a small change in the image could cause a huge change in the value of the cost function. More importantly, as $\epsilon$ increases, the Lipschitz constant of $\log _{\epsilon}$ decreases and fewer iterations are required for convergence. Figure 6 shows that with $\epsilon=0$, the cost function does not reach the limit (not flat) in 100 iterations and is not as stable as the others. A tradeoff between image sparsity and numerical stability is provided for $\epsilon$ in the range [0.001,0.004].

\footnotetext{
${ }^{1}$ With 25 IRLS iterations, the output image is not so smooth and exhibits more (boundary) artifacts.
} 

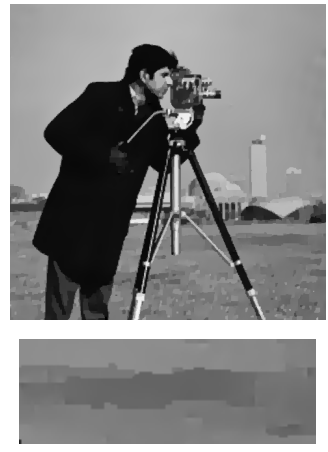

(a)
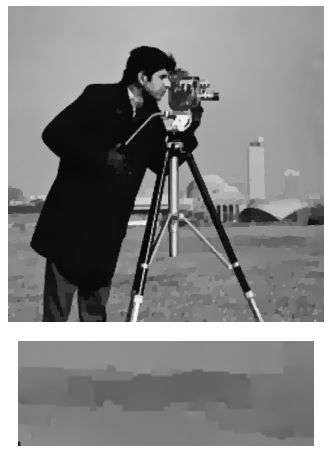

(b)
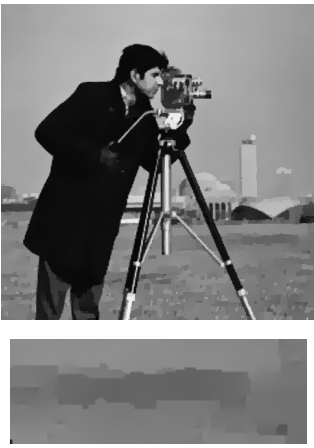

(c)
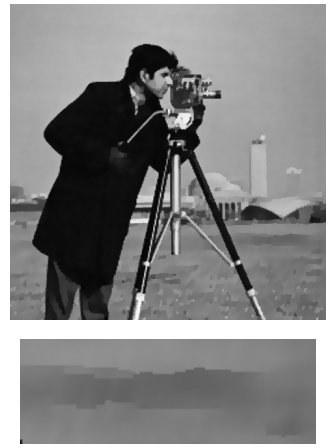

(d)
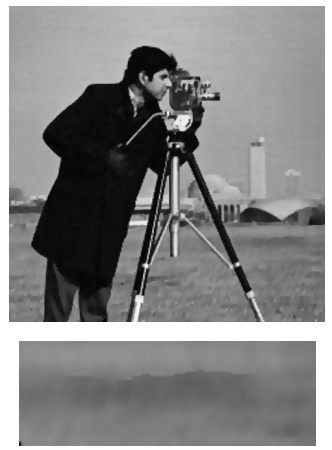

(e)

Fig. 5. Restorations with different $\epsilon$ : (a) $\epsilon=0$; (b) $\epsilon=0.002$; (c) $\epsilon=0.004$; (d) $\epsilon=0.01$; (e) $\epsilon=0.02$. Increasing $\epsilon$ makes weak edges less steep and more blurry, as shown in close-up views in the 2 nd row.

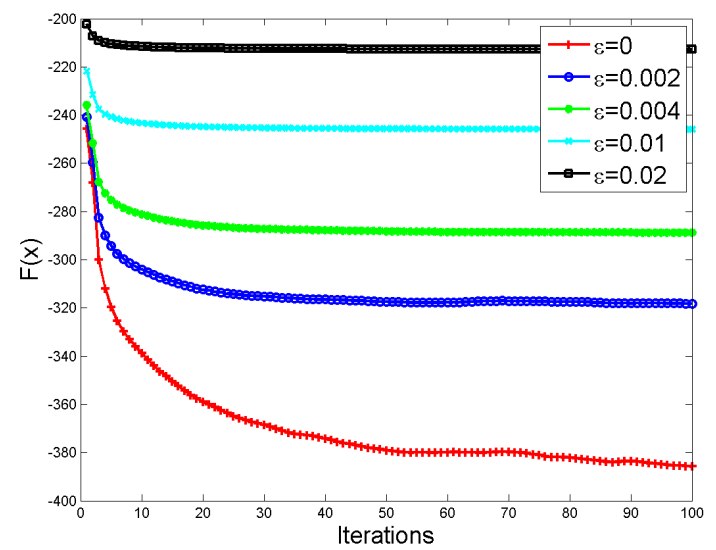

Fig. 6. Cost profiles with different values of $\epsilon$. Increasing $\epsilon$ leads to better convergence and numerical stability.

The noise variance $\sigma^{2}$ controls the tradeoff between image details and smoothness. Increasing $\sigma^{2}$ leads to larger smoothness and fewer details. For blur estimation, it is helpful to start with a large $\sigma^{2}$ in which case the blur kernel is not accurate, and then gradually decrease it to the desirable value as the kernel estimate improves [10] [13] [27]. The rationale behind this strategy is that a very smooth image can facilitate the quick identification of the blur and hence speed up convergence. In our implementation, we adapt $\sigma$ only in the first 5 iterations and then keep it fixed to 0.01 , similarly to [27].

\section{EXPERIMENTS}

We first use synthetic data to demonstrate that the proposed EM-SADMM approach is more robust than EMADMM, then we compare our EM-SADMM BID method with state-of-the-art methods, [13] [14] [25] [27]. For simplicity, we denote the proposed BID methods by the form log+ADMM or log+SADMM, where log+ADMM means Huber log prior and ADMM optimization scheme, and log+SADMM means Huber log prior and ADMM optimization scheme.

To estimate the blur kernel, only the first order derivative filters, $f_{1}=[1,-1]$ and $f_{2}=[1,-1]^{T}$, are used. For the final image reconstruction, we adopt not only the first order but also the second order derivative filters, i.e., $f_{3}=[-1,2,-1], f_{4}=[-1,2,-1]^{T}, f_{5}=[1-1 ;-1,1]$. For fair comparison, we use our fast nonblind deconvolution algorithm ${ }^{2}$ with the same parameters to recover the final image for all the BID methods, that is, to solve (31). All experiments are carried out using MATLAB on a PC with the Intel Core i7-4790 CPU @ 3.60 GHz.

\footnotetext{
${ }^{2}$ Code available at http://decsai.ugr.es/vip/resources/VDBKE.html
} 
TABLE I

QUANTITATIVE COMPARISON ON OUR TEST SET OF 40 IMAGES.

\begin{tabular}{|l|c|c|c|c|c|c|c|}
\hline Methods & PSNR & SSIM & SSD & Error Ratio & Time & $R(\hat{x})$ & $F(\hat{x})$ \\
\hline With Known $h$ & 32.90 & 0.8668 & 105.76 & 1 & - & - & - \\
$\log _{0.001}+$ ADMM & 30.05 & 0.8529 & 167.20 & 1.5825 & 25.21 & -312.62 & -310.03 \\
$\log _{0.002}+$ ADMM & 30.04 & 0.8528 & 167.23 & 1.5832 & 25.61 & -302.66 & -300.07 \\
$\log _{0.004}+$ ADMM & 30.09 & 0.8531 & 167.80 & 1.5914 & 25.51 & -285.95 & -283.37 \\
$\log _{0.01}+$ ADMM & 30.00 & 0.8507 & 172.95 & 1.6334 & 25.39 & -253.07 & -250.52 \\
$\log _{0.001}+$ SADMM & 30.10 & 0.8600 & 128.49 & 1.2049 & 16.64 & -339.52 & -336.72 \\
$\log _{0.002}+$ SADMM & 30.14 & 0.8598 & 130.04 & 1.2235 & 16.12 & -319.40 & -316.61 \\
$\log _{0.004}+$ SADMM & 30.24 & 0.8600 & 128.55 & 1.2034 & 14.80 & -294.14 & -291.38 \\
$\log _{0.01}+$ SADMM & 30.08 & 0.8535 & 158.15 & 1.5040 & 9.88 & -254.69 & -252.01 \\
\hline
\end{tabular}
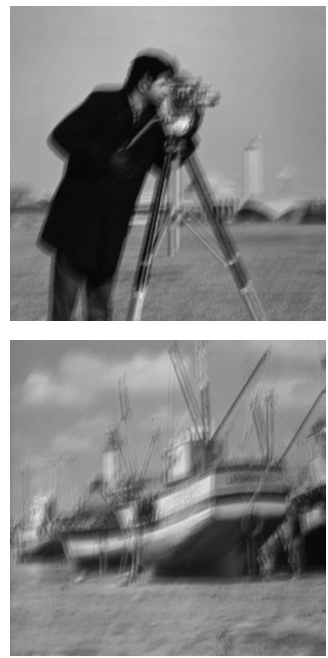

(a)
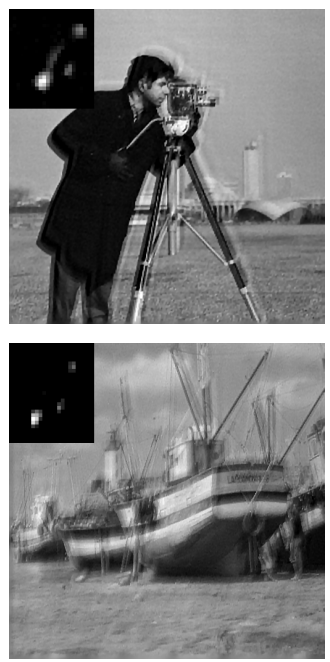

(b)
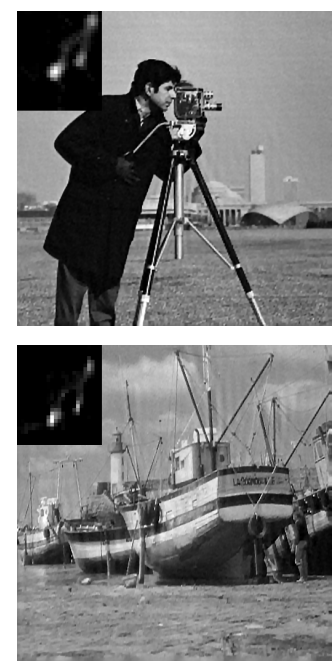

(c)
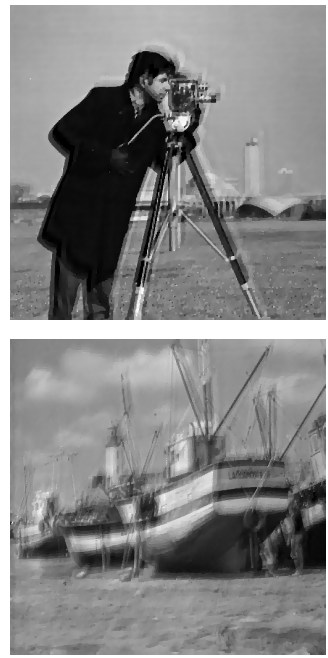

(d)
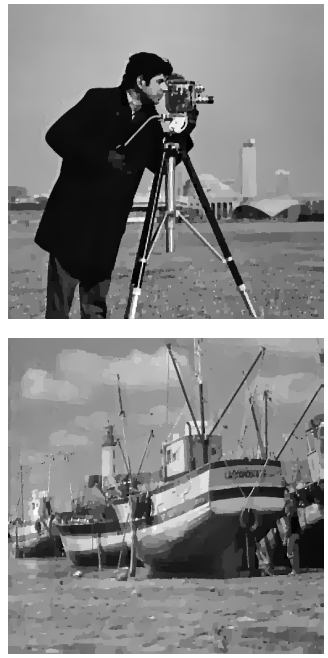

(e)

Fig. 7. The two worst deblurred results on the 40 dataset. (a) Input. (b) $x^{*}$ (see Eq. (31)) with the kernel estimated by log+ADMM. (c) $x^{*}$ with the kernel estimated by log+SADMM. (d) The estimate $\hat{x}$ by log+ADMM, see Eq. (17), Cameraman: $F(\hat{x})=-318.35, R(\hat{x})=-320.89$; Boat: $F(\hat{x})=-295.14, R(\hat{x})=-297.65$. (e) The estimate $\hat{x}$ by $\log +$ SADMM, Cameraman: $F(\hat{x})=-352.34, R(\hat{x})=-355.10$; Boat: $F(\hat{x})=-331.61, R(\hat{x})=-334.54$.

\section{A. EM-SADMM vs. EM-ADMM in blind deconvolution}

We first show that the proposed EM-SADMM approach outperforms the EM-ADMM approach in terms of restoration quality, speed, and objective function value. We choose the 5 standard images (all of size $512 \times 512$ ): Cameraman, Lena, Boat, Airplane, and Pepper, and use the 8 motion kernels in [35] to convolve each image and add $1 \%$ Gaussian noise as in [14]. Subsequently, we obtain a dataset of 40 degraded images. Table I shows the average PSNR (the highest PSNR after a shift operation, see Eq. (12) in [19]), SSIM [36], SSD (Sum of Squared Differences [35]) error, error ratio (ratio between the SSD restoration error with the estimated kernel and the SSD restoration error with the groundtruth kernel), running time, regularization $R(\hat{x})$ (smaller indicates smoother), and objective $F(\hat{x})$ (smaller is better). It is clear that the EM-SADMM approach yields better results than the EMADMM approach for all figures of merit and also is about 1.5 times faster. In addition, it shows that $\epsilon=0.004$ leads to the best performance in terms of the 4 figures of merits and running time. However, further increasing $\epsilon$ makes the overall performance worse.

The EM-SADMM approach leads to better BID performance than the EM-ADMM approach, mainly because the EM-SADMM approach better avoids undesirable saddle point and local minima than the EM-ADMM approach, as we have shown in Fig. 2. To further illustrate this point, we present the worst two EM-ADMM $(\epsilon=0.001)$ restorations in Fig. 7(b) and the corresponding image estimates $\hat{x}$ (see Eq. (17)) in Fig. 7(d). The input images are shown in Fig. 7(a). As we can see from Fig. 7(b) and (d), it is conceivable that the ringing artifacts and blur in Fig. 7.b) are indeed caused by the ringing artifacts and blur in the image estimates $\hat{x}$ in Fig. 7(d), which are typical bad 
TABLE II

RUNNING TIME (IN SECONDS) OF DIFFERENT METHODS.

\begin{tabular}{|l|l|l|l|}
\hline Kernel Support & $11 \times 11$ & $21 \times 21$ & $27 \times 27$ \\
\hline Babacan et al. $\mid$ 13] (MATLAB) & 116.48 & 302.85 & 535.71 \\
Levin et al. [10] (MATLAB) & 111.75 & 240.72 & 425.58 \\
Perrone\&Favaro [25] (MATLAB) & 2382.85 & 3447.20 & 3745.64 \\
Xu et al. [17] (C++) & 2.24 & 3.13 & 2.93 \\
$\log +$ SADMM (MATLAB) & 9.45 & 15.6 & 15.12 \\
\hline
\end{tabular}

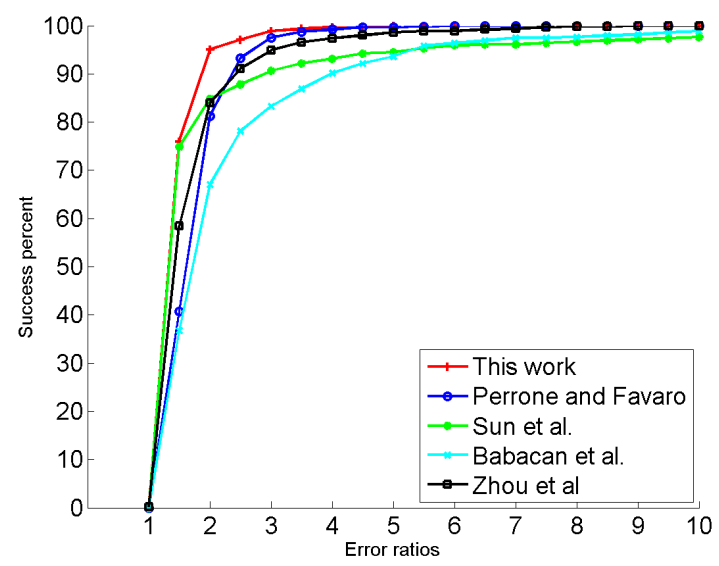

Fig. 8. Cumulative histograms of the error ratios across the dataset [14].

local minima or saddle point since the cost function $F(x)$ is not small (see the caption in Fig. 77). Interestingly, by calculating $F(\hat{x})-R(\hat{x})$, we emphasize that the image and kernel estimates obtained by log+ADMM better fit the observation data than the estimates obtained by log+SADMM, although the image and kernel estimates obtained by $\log +\mathrm{ADMM}$ are less accurate. Take the Boat image in Fig. 7 as an example; the data fitting term for $\log +\mathrm{ADMM}$ is about 2.41, smaller than 2.76, the data fitting term for log+SADMM. Therefore, we can conclude that the image and kernel estimates by log+ADMM in Fig. 7 are indeed poor stationary points. By enforcing more smoothness during the optimization process, the log+SADMM approach leads to smoother image estimates and hence is more likely to avoid ringing and blur, as shown in Fig. 7(c) and (e).

Table III shows the running time of the 5 methods to estimate three kernels of different support from a $512 \times 512$ image. As we can see, the proposed method $\log +$ SADMM with $\epsilon=0.002$ is about $10-20$ times faster than [10] and [13], depending on the kernel size. Thanks to the use of 2-D FFT, the running time of the proposed method does not increase much as the kernel gets larger, whereas both [10] and [13] become very time consuming as the kernel size increases. Due to the log prior intrinsic computation cost and the MATLAB implementation, the proposed algorithm is still much slower than the MAP method [17]. But, we argue that the BID method [17] is an "early stop" algorithm and can not achieve current state-of-the-art performance, as shown in [27]. The computational complexity of the proposed algorithm is $O\left(N_{F} N \log N\right)$, similar to [17], where $N_{F}$ denotes the number of FFTs and IFFTs, since the computations for the image and kernel estimation are both dominated by FFTs and IFFTs. In contrast, the computational complexity of the methods in [10] [13] [25] is of the form $O\left(N_{C} N K\right)$, with $N_{C}$ being the number of 2-D spatial convolutions, since 2-D convolutions dominate the computations. Notice that we have used here $K$, the blur size, instead of the image size, $N$. In general, due to the use of ADMM, $N_{F}$ can be 3-5 times smaller than $N_{C}$.

\section{B. Comparison on the Sun et al. [14] dataset}

We test the proposed methods on the dataset in [14], which contains 640 blurry and noisy images $(\sigma=0.01)$ generated from 80 natural images and 8 motion kernels. For fair comparison, we use the nonblind deconvolution method EPLL [37] to reconstruct the final restoration. Figure 8 shows the success percent of 5 methods, including the VB methods (the proposed $\log +\mathrm{SADMM}$ and Babacan et al. [13]) and the MAP methods Sun et al. [14], 


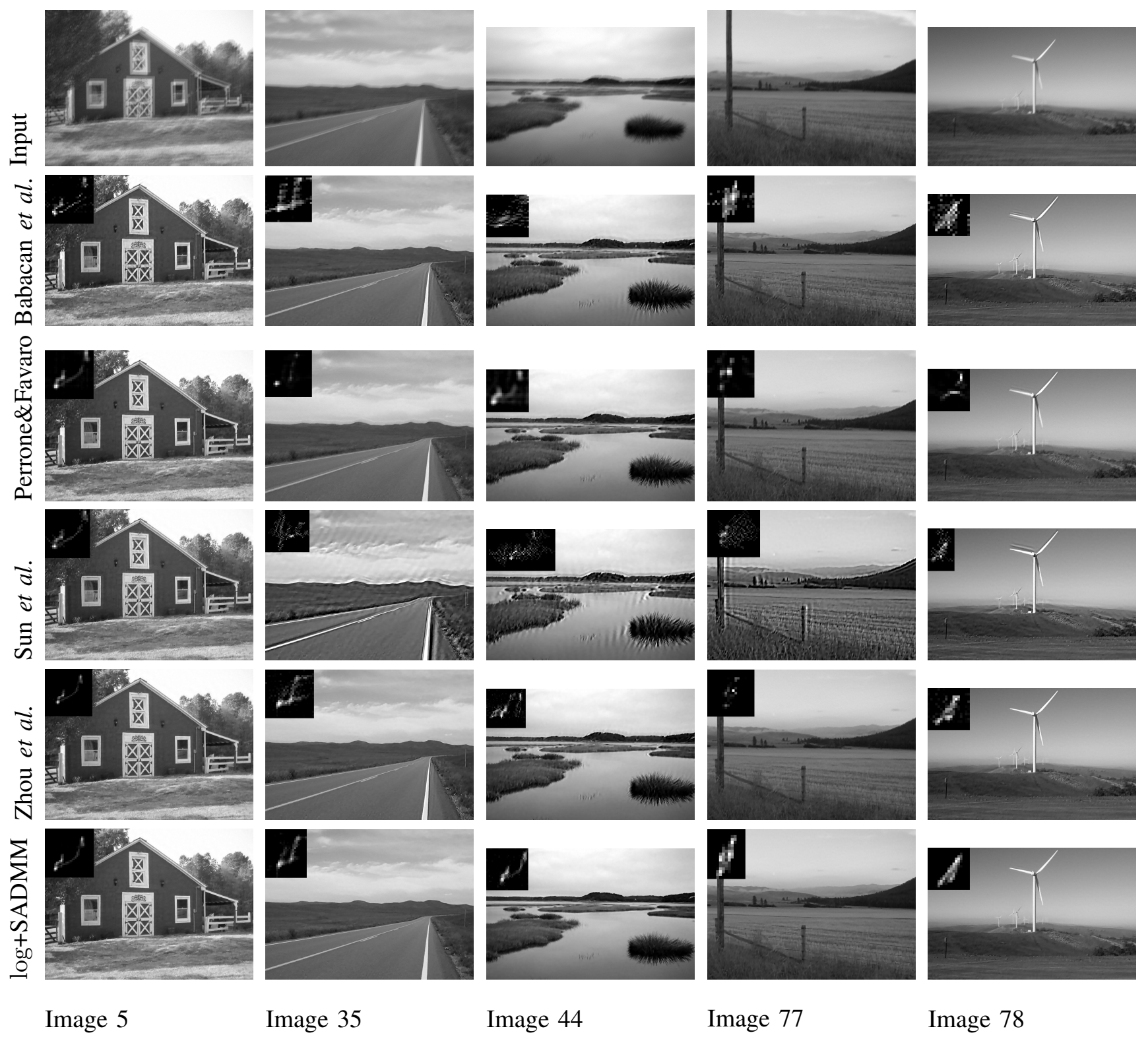

Fig. 9. Selected results on the dataset [14] for visual comparison. This figure is best viewed on the screen. Please zoom in for more details.

Perrone and Favaro [25] and Zhou et al. [27]. We observe that a restoration with error ratio less than 2 is visually satisfying (without noticeable ringing artifacts and blur). Figure 8 suggests that our method significantly outperforms the competitors, with over $75 \%$ excellent restorations (error ratio $<1.5$ ), $95 \%$ successful restorations (error ratio $<2$ ), and $100 \%$ acceptable restorations (error ratio < 5). Sun et al. [14] can also achieve about $75 \%$ excellent restorations, but is not so robust as Perrone and Favaro [25] and Zhou et al. [27], which have over 99\% and 98\% acceptable restorations, respectively. Table III shows the average SSD error and error ratio of those methods. It is clear that the proposed method takes the lead, with an average error ratio of 1.41 and SSD error of 590.

Figure 9 shows 5 selected images for visual comparison, in which only image 5 contains adequate step edges and the rest lack step edges. As we can see from Fig. 9, the step edge based method [14] works well for image 5, but fails for the other natural images. The algorithm by Babacan et al. [13] works well for image 5 but generates noticeable ringing artifacts for the rest. Our method significantly outperforms Babacan et al. [13] mainly because the proposed EM-SADMM approach better avoids poor stationary points than the EM approach, which uses CG or ADMM to solve (17) fully and cannot guarantee a smooth image estimate as SADMM does. Perrone and Favaro [25] and Zhou et al. [27] yield good restorations but still exhibiting visible ringing artifacts or blur, and the estimated kernels are not as accurate as ours, see image 44 for an example. The proposed method shows superior performance 
TABLE III

AVERAGE SCORES OF DIFFERENT METHODS.

\begin{tabular}{|l|l|l|}
\hline Methods & SSD Error & Erorr Ratio \\
\hline With Known $h$ & 392.15 & 1 \\
log+SADMM & 590.39 & 1.41 \\
Perrone \& Favaro [25] & 635.27 & 1.71 \\
Sun et al. [14] & 618.92 & 2.01 \\
Babacan et al. $[13]$ & 792.27 & 2.27 \\
Zhou et al. |27| & 686.29 & 1.66 \\
\hline
\end{tabular}

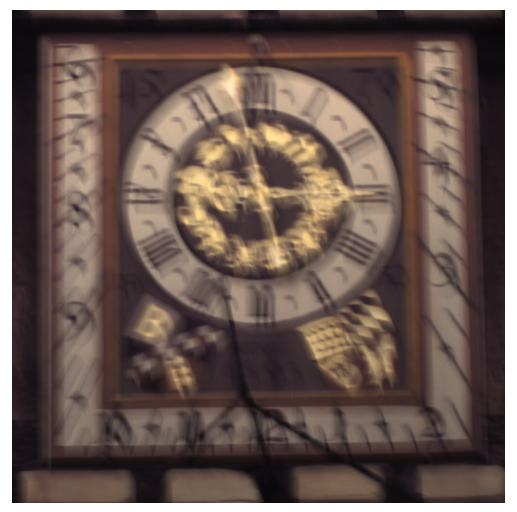

(a)

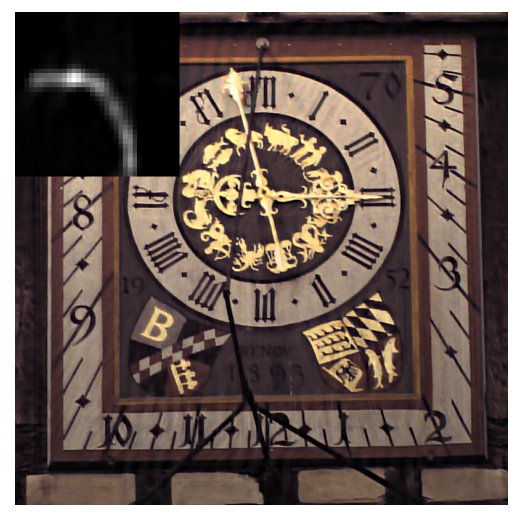

(d)

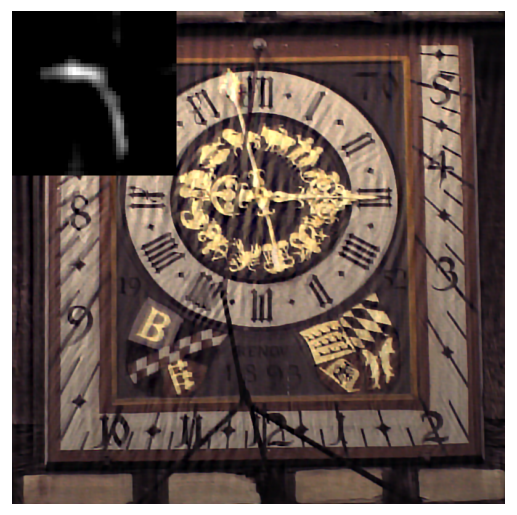

(b)

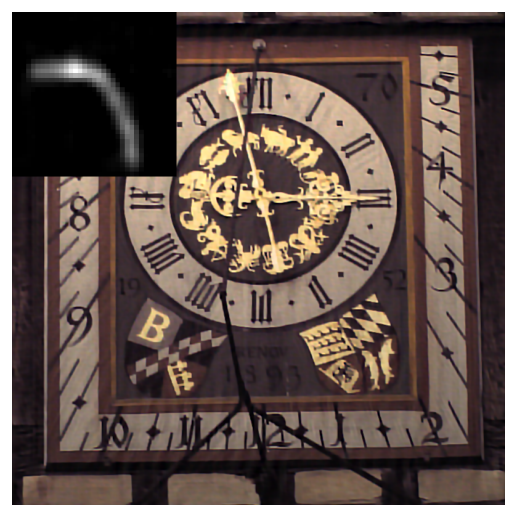

(e)

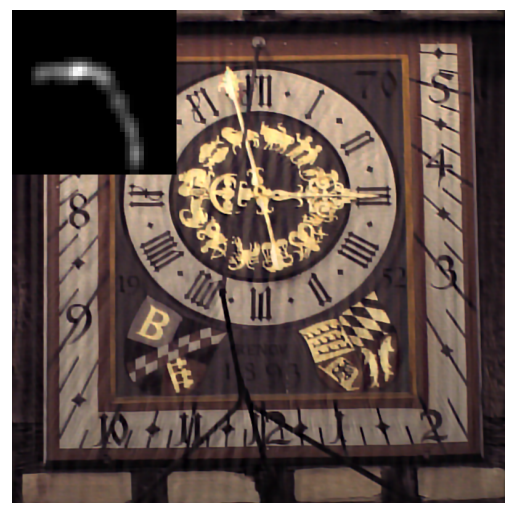

(c)

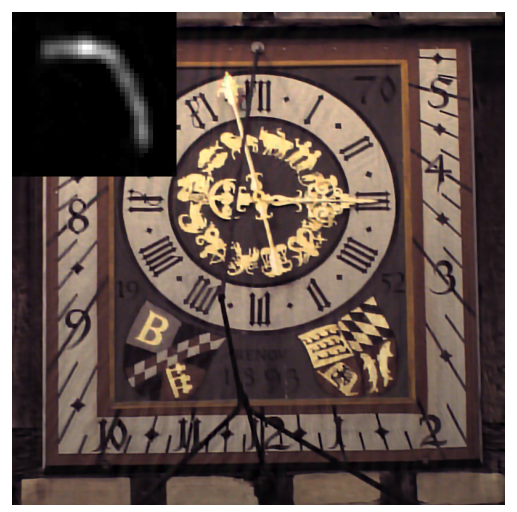

(f)

Fig. 10. Removing camera shake. (a) Input image, borrowed from [38]. (b) Babacan et al. [13]. (c) Xu et al. [17]. (d) Perrone and Favaro [25]. (e) Zhou et al. [27]. (f) The proposed log+SADMM.

for all these challenging images, without any visible ringing artifacts in the deblurred results.

\section{Results on real data}

Figure 10.a) shows a real image with blur caused by camera shake [38]. Fig. [10(b)-(f) show the deblurred images and estimated kernels by various methods. As we can see in Fig. 10, noticeable ringing artifacts emerge in the results of [13] and $\mathrm{Xu}$ et al. [17], whereas fewer ringing artifacts appear in the results of Perrone and Favaro [25], Zhou et al. [27], and ours.

\section{CONCLUSION}

In this paper, we have proposed first a new family of sparsity promoting priors, the so called Huber Super Gaussian priors, to address the numerical problem caused by the singularity at zero of some SG priors, and introduced an smooth optimization approach to deal with the poor stationary point problem caused by the use of nonconvex image priors. We show that the proposed EM-SADMM approach, which finds a smooth image mean rather than 
the exact image mean provided by the EM approach, better avoids undesirable saddle point and local minima. By making use of ADMM, a fast EM based BID method formulated in image space for these priors has been proposed. Experiments demonstrate that the proposed log+SADMM BID method significantly outperforms current state-of-the-art BID algorithms in terms of restoration quality and speed.

\section{REFERENCES}

[1] M. R. Banham and A. Katsaggelos, "Digital image restoration," IEEE Signal Process. Mag., vol. 14, no. 2, pp. $24-41,1997$.

[2] P. Ruiz, X. Zhou, J. Mateos, R. Molina, and A. K. Katsaggelos, "Variational bayesian blind image deconvolution: A review," Digital Signal Processing, vol. 47, pp. 116 - 127, 2015.

[3] J. Miskin and D. MacKay, "Ensemble learning for blind image separation and deconvolution," in Advances in Independent Component Analysis. Springer, 2000.

[4] C. Likas and N. Galatsanos, "A variational approach for bayesian blind image deconvolution," IEEE Trans. Signal Process., vol. 52, no. 8, pp. 2222-2233, Aug 2004.

[5] R. Fergus, B. Singh, A. Hertzmann, S. T. Roweis, and W. T. Freeman, "Removing camera shake from a single photograph," ACM Trans. Graph, vol. 25, no. 3, 2006.

[6] O. Shacham, O. Haik, and Y. Yitzhaky, "Blind restoration of atmospherically degraded images by automatic best step-edge detection," Pattern Recognition Letters, vol. 28, no. 15, pp. 2094-2103, 2007.

[7] J. H. Money and S. H. Kang, "Total variation minimizing blind deconvolution with shock filter reference," Image and Vision Computing, vol. 26, no. 2, pp. 302-314, 2008.

[8] S. Cho and S. Lee, "Fast motion deblurring," ACM Trans. Graph, vol. 28, no. 5, 2009.

[9] J. Cai, H. Ji, C. Liu, and Z. Shen, "Blind motion deblurring from a single image using sparse approximation," in CVPR. IEEE, 2009.

[10] A. Levin, Y. Weiss, F. Durand, and W. T. Freeman, "Efficient marginal likelihood optimization in blind deconvolution," in CVPR. IEEE, 2011

[11] D. Krishnan, T. Tay, and R. Fergus, "Blind deconvolution using a normalized sparsity measure," in CVPR. IEEE, 2011.

[12] A. Goldstein and R. Fattal, "Blur-kernel estimation from spectral irregularities," in ECCV, 2012.

[13] S. Babacan, R. Molina, M. Do, and A. Katsaggelos, "Bayesian blind deconvolution with general sparse image priors," in ECCV, 2012.

[14] L. Sun, S. Cho, J. Wang, and J. Hays, "Edge-based blur kernel estimation using patch priors," in ICCP. IEEE, 2013.

[15] L. Zhong, S. Cho, D. Metaxas, S. Paris, and J. Wang, "Handling noise in single image deblurring using directional filters," in CVPR. IEEE, 2013.

[16] L. Xu and J. Y. Jia, "Two-phase kernel estimation for robust motion deblurring," in ECCV, 2010.

[17] L. Xu, S. C. Zheng, and J. Y. Jia, "Unnatural 10 sparse representation for natural image deblurring," in CVPR. IEEE, 2013.

[18] M. S. C. Almeida and M. A. T. Figueiredo, "Blind image deblurring with unknown boundaries using the alternating direction method of multipliers," in ICIP. IEEE, 2013.

[19] X. Zhou, F. Zhou, and X. Bai, "Blind deconvolution using a nondimensional gaussianity measure," in ICIP. IEEE, 2013.

[20] H. Zhang and D. Wipf, "Non-uniform camera shake removal using a spatially-adaptive sparse penalty," in NIPS, 2013.

[21] M. Vega, R. Molina, and A. Katsaggelos, "Parameter estimation in bayesian blind deconvolution with super gaussian image priors," in EUSIPCO, 2014.

[22] R. Molina, J. Mateos, and A. Katsaggelos, "Blind deconvolution using a variational approach to parameter, image, and blur estimation," IEEE Trans. Image Process., vol. 15, no. 12, pp. 3715-3727, Dec 2006.

[23] D. Wipf and H. Zhang, "Revisiting bayesian blind deconvolution," Journal of Machine Learning Research, vol. 15, no. 1, pp. 3595-3634, 2014.

[24] S. Osher and L. I. Rudin, "Feature-oriented image enhancement using shock filters," SIAM Journal on Numerical Analysis, vol. 27, no. 4, pp. 919-940, 1990.

[25] D. Perrone and P. Favaro, “A logarithmic image prior for blind deconvolution,” International Journal of Computer Vision, pp. 1-14, 2015.

[26] T. F. Chan and C.-K. Wong, "Total variation blind deconvolution," IEEE Trans. Image Process., vol. 7, no. 3, pp. 370-375, 1998.

[27] X. Zhou, J. Mateos, F. Zhou, R. Molina, and A. Katsaggelos, "Variational dirichlet blur kernel estimation," IEEE Trans. Image Process., vol. 24, no. 12, pp. 5127-5139, Dec 2015.

[28] X. Zhou, R. Molina, F. Zhou, and A. Katsaggelos, "Fast iteratively reweighted least squares for $l_{p}$ regularized image deconvolution and reconstruction," in ICIP. IEEE, 2014.

[29] R. T. Rockafellar, Convex Analysis. Princeton University Press, 1996.

[30] M. Hintermüller and T. Wu, "Nonconvex $T V^{q}$-models in image restoration: analysis and a trust-region regularization based superlinearly convergent solver," Siam Journal on Imaging Sciences, vol. 6, no. 3, pp. 1385-1415, 2013.

[31] Y. L. Wang, J. F. Yang, W. T. Yin, and Y. Zhang, "A new alternating minimization algorithm for total variation image reconstruction," SIAM Journal on Imaging Sciences, vol. 1, no. 3, pp. 248-272, 2008.

[32] A. Levin, R. Fergus, F. Durand, and W. T. Freeman, "Image and depth from a conventional camera with a coded aperture," ACM Trans. Graph, vol. 26, no. 3, 2007.

[33] X. Zhou, F. Zhou, X. Bai, and B. Xue, "A boundary condition based deconvolution framework for image deblurring," Journal of Computational and Applied Mathematics, vol. 261, pp. 14-29, 2014.

[34] S. Boyd, N. Parikh, E. Chu, B. Peleato, and J. Eckstein, "Distributed optimization and statistical learning via the alternating direction method of multipliers," Foundations and Trends in Machine Learning, vol. 3, pp. 1-122, 2011.

[35] A. Levin, Y. Weiss, F. Durand, and W. T. Freeman, "Understanding and evaluating blind deconvolution algorithms," in CVPR. IEEE, 2009. 
[36] Z. Wang, A. C. Bovik, H. R. Sheikh, and E. P. Simoncelli, "Image quality assessment: from error visibility to structural similarity," IEEE Trans. Image Process., vol. 13, no. 4, pp. 600-612, 2004.

[37] D. Zoran and Y. Weiss, "From learning models of natural image patches to whole image restoration," in ICCV. IEEE, 2011.

[38] R. Köhler, M. Hirsch, B. Mohler, B. Schölkopf, and S. Harmeling, "Recording and playback of camera shake: Benchmarking blind deconvolution with a real-world database," in ECCV, 2012. 